\title{
ANALYSIS OF INTRA-URBAN TRAFFIC ACCIDENTS USING SPATIOTEMPORAL VISUALIZATION TECHNIQUES
}

\author{
Ali Soltani $^{1,2}$, Sajad Askari ${ }^{1}$ \\ ${ }^{I}$ Department of Urban Planning, Shiraz University, Iran, Goldasht St., Maaliabad, Shiraz \\ Phone: +987116230453. E-mail: soltani@shirazu.ac.ir \\ ${ }^{2}$ Urban Research program, Griffith University, QLD, 400, Australia \\ Phone:+61737357003.E-mail: a.soltani@griffith.edu.au
}

Road traffic accidents (RTAs) rank in the top ten causes of the global burden of disease and injury, and Iran has one of the highest road traffic mortality rates in the world. This paper presents a spatiotemporal analysis of intra-urban traffic accidents data in metropolitan Shiraz, Iran during the period 2011-2012. It is tried to identify the accident prone zones and sensitive hours using Geographic Information Systems (GIS)-based spatio-temporal visualization techniques. The analysis aimed at the identification of high-rate accident locations and safety deficient area using Kernel Estimation Density (KED) method. The investigation indicates that the majority of occurrences of traffic accidents were on the main roads, which play a meta-region functional role and act as a linkage between main destinations with high trip generation rate. According to the temporal distribution of car crashes, the peak of traffic accidents incident is simultaneous with the traffic congestion peak hours on arterial roads. The accident-prone locations are mostly located in districts with higher speed and traffic volume, therefore, they should be considered as the priority investigation locations to safety promotion programs.

Keywords: Accident; Traffic; Spatio-temporal Analysis; Shiraz

\section{Introduction}

Road Traffic Accidents (RTAs) have been and are continuing to be a major contributor of human and economic costs to requiring concerted multi-disciplinary efforts for sustainable effective prevention. RTAs rank in the top ten causes of the global burden of disease and injury, and will probably be in third place by 2020 , when measured in disability-adjusted life years lost (WHO, 2013). With only 25 percent of all motorized vehicles, developing countries account for 86 percent of all road traffic deaths (Lagarde, 2011). Approximately 1.24 million people die every year on the world's roads (WHO, 2013), and the estimated costs of $\$ 518$ billion (Ivers, Stevenson, Norton, \& Yu, 2008) and another 20 to 50 million sustain non-fatal injuries as a result of RTAs. Early in this decade, some 20 to 50 million people in the world became totally or partially incapacitated due to injuries caused by traffic accidents. Injured and trauma victims took 10 per cent of all hospital beds that year (Ivers, Stevenson, Norton, \& Yu, 2008). Current trends suggest that by 2030 road traffic deaths will become the fifth leading cause of death unless urgent action is taken (WHO, 2013). A great number of patients are transported to the emergency ward due to traffic accident injuries. These cases are a daily challenge for the teams working in pre and intrahospital settings, especially due to the severity of the injuries and to the time it takes to reach the hospital and forward patients to surgery (Calil et al., 2009). Unless appropriate action is taken urgently, the problem will worsen globally. This will particularly be the case in those developing countries where rapid motorization is likely to occur over the next two decades (Mohammadi, 2013). The main reasons behind the dramatic increases of the number of RTAs can be listed as an insufficient road system, a rapid increase in the number of motorized vehicles, inadequacy of road safety policies, reckless driving and poor emergency services (Akgüngör, 2007).

Iran has one of the highest road traffic mortality rates in the world. Motor vehicle-related accidents account for more than 1'300'000 years of lost life around the country each year (Tavafian, Aghamolaei, Gregory, \& Madani, 2011). The mortality rate due to traffic injuries has become considerable particularly during the last decade along with the industrialization process (Bahadorimonfared, et al., 2013).

The primary objective of this paper is to determinate hotspots location and sensitive times for Shiraz. It provides a manifest to policies assembling and implementation plan in urban road safety planning. The rest of this paper is structured as follows. In the second section, the relevant literature and methodology are reviewed. In Section three, the case study area and traffic accidents data are described. Section four discusses estimation methodologies, and presents the empirical results. The last section concludes. 


\section{Literature Review}

RTAs result in injuries and death, but many are preventable, however they are commonly anticipated to form clusters in the geographic space and over time for the reason that their occurrence is tied to traffic volumes (Yamada \& Thill, 2004). To developing strategies to prevent them, reducing traffic accidents, and improving road safety, an imperative need to understand how, where and when RTAs occurred and distributed across space and time (Brunsdon, Cocoran, \& Higgs, 2007; Xie \& Yan, 2008; Plug, Xia, \& Caulfield, 2011), and utilize spatiotemporal patterns in road safety policy capturing. The importance of having a comprehensive crash map has been highlighted as a significant component of safety data management in strategic highway safety plans (Qin, Parker, Liu, Graettinger, \& Forde, 2013). Injuries due to RTA depend upon a number of factors-human, vehicle and environmental factors play vital roles before, during and after a serious RTA. The significant factors are human errors, driver fatigue, poor traffic sense, poor condition of vehicle, speeding and overtaking violation of traffic rules, poor road infrastructure, traffic congestion, and road encroachment. In terms of severity of accident, driving speeds are of major importance as well. Environmental factors include the condition of the road network, road type and design, spatial context (e.g. density and land-use; plantation etc.), temporal context (e.g., darkness) and transport context (traffic density, speed and behaviour of other transport users). Social and psychological factors include socio-demographic and socio-economic structures, risk attitudes, lifestyles and 'mobility styles' and associated behaviour (Holz-Rau and Scheiner, 2013).

Identifying accident hotspots and appending value added data to understand the processes happening in these hotspots are important for the appropriate allocation of resources for safety improvements. By identifying road accident, a more robust understanding can be gained, with regards to indicators of casual effects (Anderson, 2009). In order to conduct a reliable analysis of the RTAs develop control strategies, it is required to investigate firstly how the accidents are geographically distributed, secondly regions where accident is observed more dense, and thirdly their geo-statistical aspects (Ozkan, Tarhan, Eser, Yakut, \& Saygin, 2013). An improved understanding of the spatial patterns of RTAs can make accident reduction efforts more effective (Xie \& Yan, 2008). Spatiotemporal GIS analysis complements and adds value to the traditional methods of identifying accidents patterns in time and space (Asgary, Ghaffari, \& Levy, 2010). There are some important factors that may impact the distribution of traffic accidents, including natural and environmental characteristics such as physical environment (steep slope, sharp turn), weather (rain, snow, wind, and fog), configuration of highway networks such as the locations of access and egress points, deficient design and maintenance of highways, etc. All of these factors more or less are associated with distinct spatial patterns as well (Xie \& Yan, 2008).

Currently, three major spatiotemporal pattern analyses and visualization techniques have been applied in vehicle crash research: map animation, iso-surface method and co-map (Asgary, Ghaffari, \& Levy, 2010). The co-map method is useful for highlighting differences in a crash pattern using 'small multiples' of diagrams (Brunsdon, Cocoran, \& Higgs, 2007). A time period, as a third dimension, is broken down into a series of time intervals and a spatial pattern can then be analyzed and illustrated for each time interval (Plug, Xia, \& Caulfield, 2011). This paper amplified one of the visualization techniques to investigate spatiotemporal structures of RTAs. First, for spatial analysis of RTAs, the Kernel Density Estimation (KDE) in a GIS environment is used to determinate critical areas with high RTAs risk.

There are a variety of spatial tools developed to assist the understanding of the changing geographies of point patterns. The most promising of these tools is KDE. The KDE is one of the most common and well-established methods in identifying spatial patterns, and a non-parametric method that involves introducing a symmetrical surface over each point feature, assessing the distance from the point to a reference location based on a mathematical function, and subsequently, adding the value of all the surfaces for that reference location (Blazquez \& Celis, 2013). The main benefit of this approach lies in recognizing the risk spread of an accident (Anderson, 2009). KDE calculates the density of events in a neighbourhood around those events. KDE allows some events to weigh more heavily than others, depending on their meaning, or to allow one event to represent several observations (Asgary, Ghaffari, \& Levy, 2010).

The spread of risk can be defined as the area around a defined cluster in which there is an increased probability for an accident to happen based on spatial dependency. Secondly by using this density measure, an arbitrary spatial unit of analysis can be defined and be homogenous for the whole area which makes comparison and ultimately a taxonomy possible (Anderson, 2009).

KDE includes placing a symmetrical surface over each point and then measuring the distance from the point to a reference location based on a mathematical function and then summing the value for all the surfaces for that reference location. This procedure is repeated for successive points. This therefore allows us to place a Kernel over each observation, and summing these individual Kernels gives us the density estimate for the distribution of accident points (Eq. 1) (Fotheringham, Brunsdon, \& Charlton, 2000; Anderson, 2009). 
Eq. 1:

$f(x, y)=\frac{1}{n h^{2}} \sum_{i=1}^{n} k\left(\begin{array}{l}d_{i} \\ h\end{array}\right)$,

where $f(x, y)$ is the density estimate at the location $(\mathrm{x}, \mathrm{y}) ; \mathrm{n}$ is the number of observations, $\mathrm{h}$ is the bandwidth or kernel size, $\mathrm{k}$ is the kernel function, and $\mathrm{d}_{\mathrm{i}}$ is the distance between the location $(\mathrm{x}, \mathrm{y})$ and the location of the $i$ th observation. The effect of placing these humps or kernels over the points is to create a smooth and continuous surface. The method is known as KDE because around each point at which the indicator is observed a circular area (the Kernel) of defined bandwidth is created. This takes the value of the indicator at that point spread into it according to some appropriate function. Summing all of these values at all places, including those at which no incidences of the indicator variable were recorded, gives a surface of density estimates (Silverman, 1986).

Kernel Density may also be used for calculating the density of linear features in the neighbourhood of each output cell. A smoothly curved surface is conceptually fitted over each line. Its value is biggest on the line and diminishes as you move away from the line, reaching zero at the specified search radius distance from the line. The surface is defined so the volume under the surface equals the product of line length and the population field value. The density at each output raster cell is calculated by adding the values of all the kernel surfaces where they overlay the raster cell centre (Silverman, 1986).

\section{The Study Area and Data}

Metropolitan Shiraz located in the southwest of Iran and is the capital city of Fars Province. According to the report from Iranian Bureau of Statistics (IBS, 2013), the population of the metropolitan area is about 1'460'000. Due to a long historical background and rich cultural adventure, Shiraz has been one of the most attractive destinations for seasonal domestic holiday-makers. However, Shiraz has suffered from intra-urban traffic accidents due to a considerable increase of vehicle ownership and private car users (Soltani, Zargari and Esmaeili, 2013). Essentially, one of the main factors contributing to the increase in intra-urban traffic accidents is the improving of social welfare in recent years.

Intra-urban traffic accidents data were collected using the secondary database of the Road Police Department, Shiraz Branch. These data was originally recorded by police officers from March 2011 to March 2012. The information on street network was obtained in a GIS format from the Municipality of Shiraz, Department of Traffic.

Table 1 details the frequencies of different types of crash. The majority of accidents occurred as crashing a car with another car ( 73 per cent) followed by a car hitting an object ( 5 per cent) and a car hitting a pedestrian passing through (5 per cent).

Table 1. The distribution of the intra-urban traffic accidents based on the type of crash

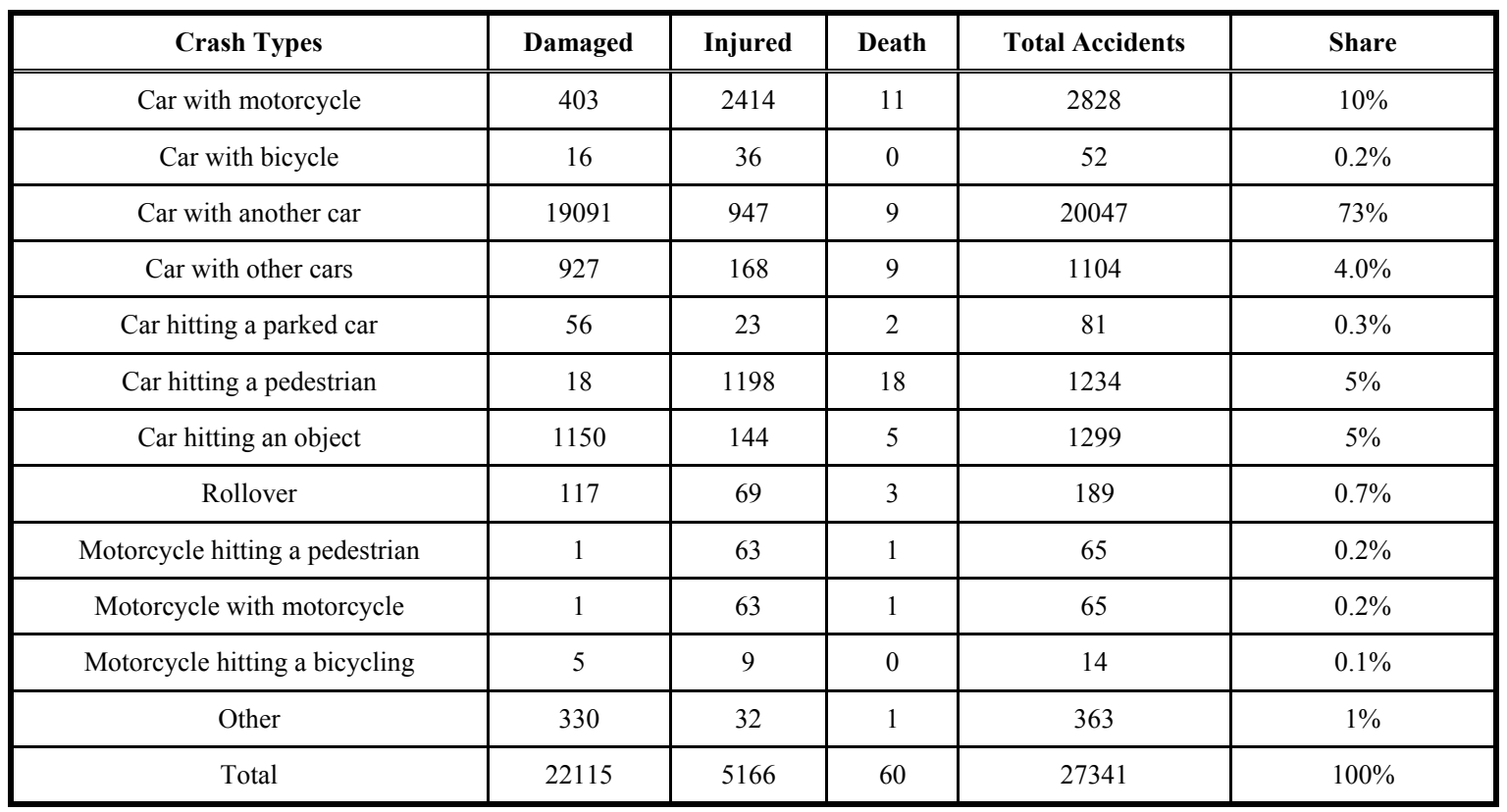




\section{Results and Discussions}

The results of this study could give an insight into the present scenario of the traffic condition of the metropolitan and shows out the most potentially accident prone roads in the district. It would also describe some causes of accident.

According to the literature of accident analysis, time is an important factor which contributes to road traffic accidents. The temporal analysis aims to discover the relative risk pattern temporally and can be used as prerequisite for spatial analysis targeting spatial patterns in different time periods

This study focuses on urban areas at a specific time of day; demonstrates spatiotemporal variations in RTAs incidents. Figure 1 illustrates how the number of RTAs across Metropolitan Shiraz areas varied from 2009 to 2010 throughout the day. This shows that a few RTAs occurred during early morning particularly they dipped between 4 and 6 AM when traffic volume is quiet low and drivers are less likelly to hit another car. On the other hand, the number of traffic accidents significantly increased after 8 AM coinciding with the beginning of activities during a typical day. This is consistent with the findings of (Heydari et al., 2013) the time distribution of fatal traffic accidents in Fars Province of Iran. They found that traffic accident deaths of students, clerks, workers and house-keepers increased dramatically at 8-12 AM.

For the spatial analysis of RTAs, this study employed KDE method to generate maps to determining RTA hotspots in Shiraz applying linear analysis supported by GIS. The Spatial Analyst KDE tool in ESRI's ArcGIS 9.3 software was used to calculate the KDE. Figure 2 shows RTAs incident patterns broken down by the day of the week. According to the spider plot, RTAs have uniform patterns during the weekdays. Figure 3 depicts the stable patterns when the month of the year is used. There is significant issue in monthly distribution that RTAs peak off period occurred in summer months and early fall months in Shiraz. As previously mentioned, this may be explained by the presence of tourists in the city because good weather conditions allow people to come to Shiraz in summer more frequently than other times of the year.

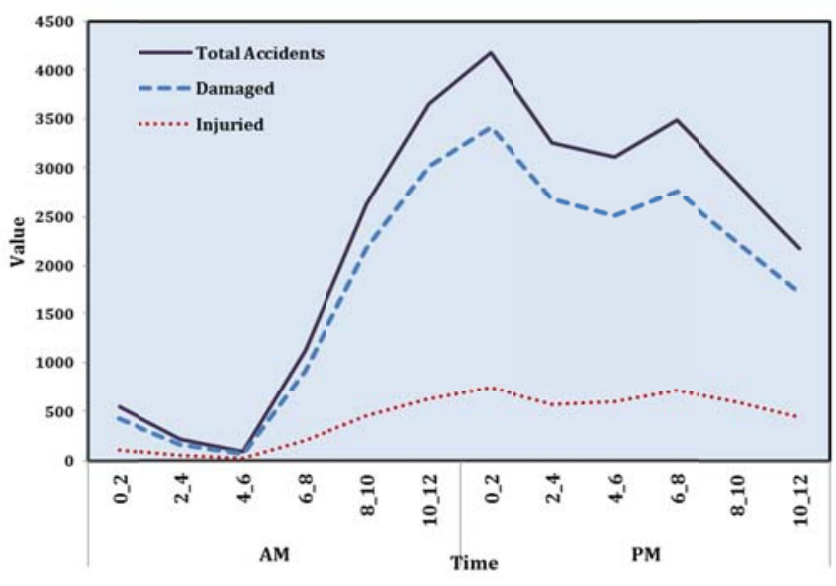

Figure 1. Hourly patterns of RTAs incidents in Shiraz, 2009-2010

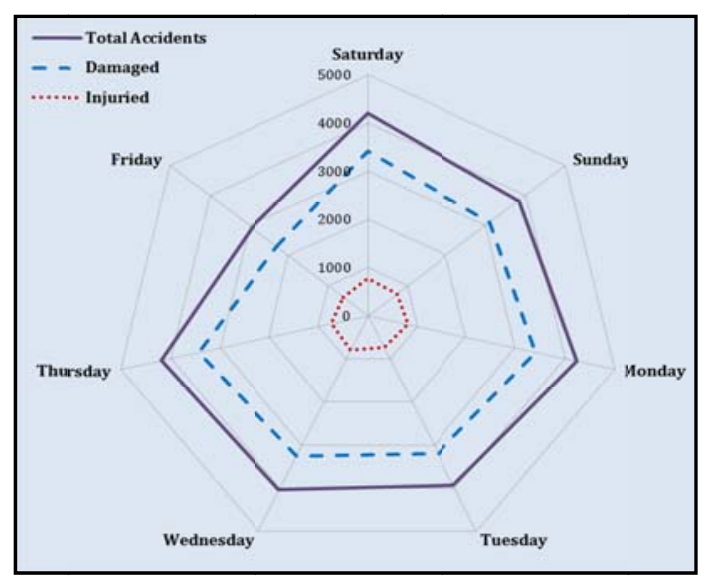

Figure 2. Patterns by day of the week 


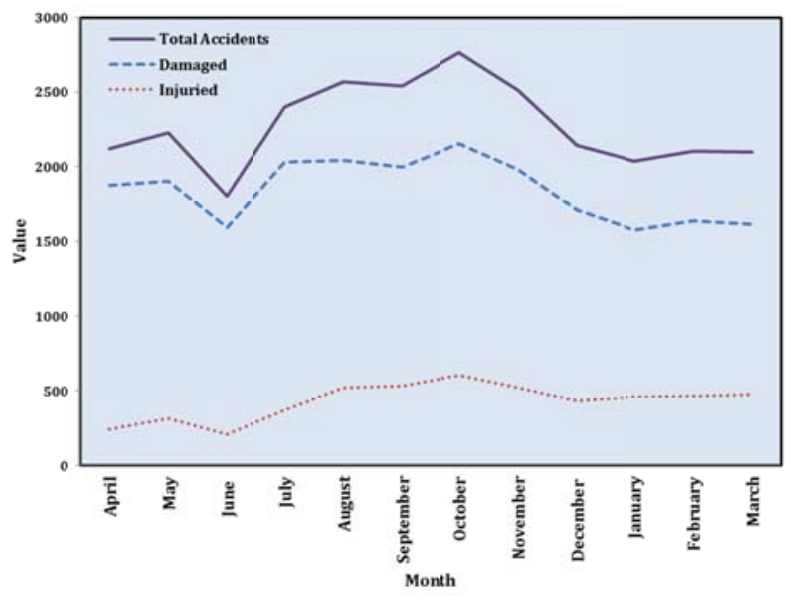

Figure 3. RTAs patterns by month of the year

Figure 4 displays the results the KDE analysis for RTAs during the study period in Shiraz. Like the temporal patterns, the spatial patterns of RTAs incidents of different causes show some variations across the metropolitan regions. RTAs incidents were more frequent in main arteries where more urban activities are taking place and clustered in the areas of urban that have high traffic volume and provided more accessibility to other road. There are a few hotspots in downtown area and some on the major streets and roads in the North-western and South-eastern parts, of the metropolitan. This map highlights, the highest RTAs incident intensity is found in the North-western parts of the metropolitan. These areas represent neighbourhoods with low population densities, newer developments, and higher quality.

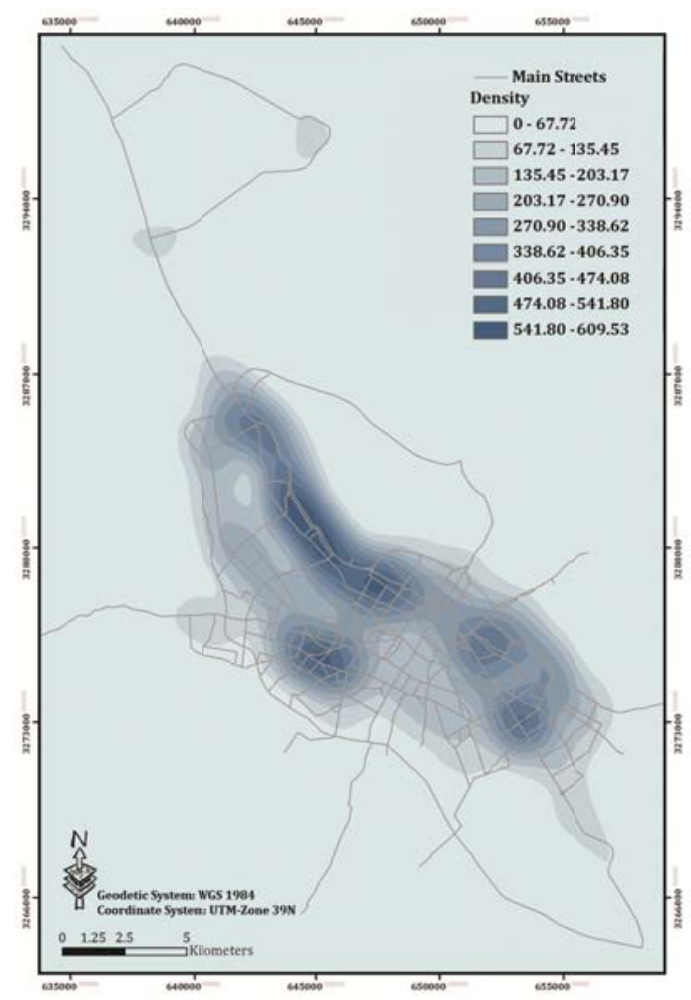

Figure 4. Intra-urban traffic accidents density using $2 \mathrm{~km}$ bandwidth of main streets

\section{Conclusions}

The analysis of spatiotemporal distribution of accidents in Metropolitan Shiraz, focuses on the total number of accidents as well as accidents happened during night and day hours (temporal) and accidents in areas with high traffic congestion (spatial). The advantages of such two dimensional hotspot surface representations, especially on intra-urban traffic accidents, can provide a more realistic 
continuous model of accident hotspot patterns, over space and time. The findings of this paper propose that RTAs trends differ according to incident type and can provide important exploratory analysis prior to more detailed investigations including the use of contextual data (e.g., land use, type of road). The results of this paper presumably have important implications for RTAs prevention planning, and RTAs intervention policies, including the identification of hotspots, which geographically define acute problem areas. Within these hotspots, traffic and police departments could focus on regions that are statistically likely to have higher levels of the repeated road traffic accidents. This study can be developed further by investigating the potential causes of traffic occurrence throughout the metropolitan area.

\section{References}

1. Akgüngör, A. (2007) Road traffic accidents and safety programme in Turkey. International Journal of Injury Control and Safety Promotion, 14(2), 119-121.

2. Anderson, T.K. (2009). Kernel density estimation and K-means clustering to profile road accident hotspots. Accident Analysis and Prevention, 41(3), 359-364.

3. Asgary, A., Ghaffari, A. \& Levy, J. (2010) Spatial and temporal analyses of structural fire incidents and their causes: A case of Toronto, Canada. Fire Safety Journal, 45(1), 44-57.

4. Bahadorimonfared, A., Soori, H., Mehrabi, Y., Delpisheh, A., Esmaili, A., Salehi, M. et al. (2013) Trends of Fatal Road Traffic Injuries in Iran (2004-2011). PLOS ONE, 8(5: e65198).

5. Blazquez , C. \& Celis, M. (2013) A spatial and temporal analysis of child pedestrian crashes in Santiago, Chile. Accident Analysis \& Prevention, 50, 304-311.

6. Brunsdon, C., Cocoran, J. \& Higgs, G. (2007) Visualising space and time in crime patterns: A comparison of methods. Computers, Environment and Urban Systems, 31(1), 52-75.

7. CalilI, A., Elias, M., Sallum, A., Alencar, C., Nogueira, S. (2009) Mapping injuries in traffic accident victims: a literature review, Review of Latino-America, Enfermagem, 17 (1).

8. Fotheringham, A., Brunsdon, C. \& Charlton, M. (2000) Quantitative Geography: Perspectives on Spatial Data Analysis. Sage Publications, Ltd.

9. Heydari, S., Hoseinzadeh, A., Sarikhani, Y., Arya, H., Mohammad, Z., Ghasem, M. et al. (2013) Time analysis of fatal traffic accidents in Fars Province of Iran. Chinese Journal of Traumatology, 16(2), 84-88.

10. Holz-Rau, C. and Scheiner, J. (2013) Geographical Patterns in Road Safety: Literature Review and a Case Study from Germany, European Journal of Transport and Infrastructure Research 13(2), 99-122.

11. Ivers, R., Stevenson, M., Norton, R. \& Yu, J. (2008) Road Traffic Injuries. International Encyclopedia of Public Health, 615-623.

12. Lagarde, E. (2011) Road Traffic Injuries. Encyclopedia of Environmental Health, 892-900.

13. Mohammadi, G. (2013) Road traffic crash injuries and fatalities in the city of Kerman, Iran. International Journal of Injury Control and Safety Promotion, 20(2), 184-191.

14. Ozkan, S., Tarhan, C., Eser, S., Yakut, C. \& Saygin, O. (2013) Spatial point pattern analysis of lung cancer in an urban area. In C. Ellul, S. Zlatanov, M. Rumor, \& R. Laurini (Eds.), Urban and Regional Data Management (pp. 77-88). London: CRC Press.

15. Plug, C., Xia, J. \& Caulfield, C. (2011) Spatial and temporal visualisation techniques for crash analysis. Accident Analysis and Prevention, 43(6), 1937-1946.

16. Prasannakumar, V., Vijith, H., Charutha, R., \& Geetha, N. (2011) Spatio-Temporal Clustering of Road Accidents: GIS Based Analysis and Assessment. International Conference: Spatial Thinking and Geographic Information Sciences. 21 (pp. 317-325). Procedia - Social and Behavioral Sciences.

17. Qin, X., Parker, S., Liu, Y., Graettinger, A. \& Forde, S. (2013) Intelligent geocoding system to locate traffic crashe. Accident Analysis \& Prevention, 50, 1034-1041.

18. Silverman, B. (1986) Density Estimation for Statistics and Data Analysis. New York: Chapman and Hall/CRC.

19. Soltani, A., Zargari Marandi, E. \& Esmaili Ivaki, Y. (2013) Bus route evaluation using a two-stage hybrid model of Fuzzy AHP and TOPSIS. Journal of Transport Literature, 12(2), 149-158.

20. Tavafian, S., Aghamolaei, T., Gregory, D. \& Madani, A. (2011) Prediction of Seat Belt Use Among Iranian Automobile Drivers: Application of the Theory of Planned Behavior and the Health Belief Model. Traffic Injury Prevention, 12(1), 48-53.

21. Vakili, V., Danaei, M., Askarian, M., Palenik, C.J. \& Abdollahifard, G. (2012) Transportation Behaviors in Shiraz, Iran. Traffic Injury Prevention, 13(3), 293-299.

22. WHO (World Health Organization). (2013) Global status report on road safety 2013: supporting a decade of action. Geneva: World Health Organization.

23. Xie, Z. \& Yan, J. (2008) Kernel Density Estimation of traffic accidents in a network space. Computers, Environment and Urban Systems, 32(5), 396-406. 